International Journal of Pure and Applied Mathematics

Volume 105 No. 2 2015, 163-172

ISSN: 1311-8080 (printed version); ISSN: 1314-3395 (on-line version)

url: http://www.ijpam.eu

doi: http://dx.doi.org/10.12732/ijpam.v105i2.4

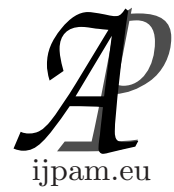

\title{
A CONTRIBUTION TO THE STUDY OF THE PROBABILISTIC HAUSDORFF METRIC
}

\author{
Abderrahim Mbarki ${ }^{1}$, Rachid Naciri ${ }^{2}$ \\ ${ }^{1}$ national School of Applied Sciences \\ P.O. Box 669, Oujda University \\ Matsi Laboratory, MOROCCO \\ ${ }^{2}$ Matsi Laboratory \\ Oujda University \\ 60000, Oujda, MOROCCO
}

\begin{abstract}
The purpose of the present paper is to study several important properties of the probabilistic Hausdorff metric. Indeed, we prove that the collection of nonempty compact subsets of a probabilistic metric space is complete with respect to the probabilistic Hausdorff metric if and only if the probabilistic metric space is complete. As a consequence, the relationship between completion of the probabilistic Hausdorff metric for a given PM space and probabilistic Hausdorff metric of its completion is examined. Finally, Deterministic counterpart of these results is deduced. Our work extend some results of [2] and [12].
\end{abstract}

AMS Subject Classification: 54E70, 54A40, 54B20

Key Words: probabilistic Hausdorff metric, complete, isometric

\section{Introduction and Preliminaries}

The concept of probabilistic metric space was first introduced and studied in 1942 by K.Menger [7]. It is a probabilistic generalization of metric space in which the distance $d(x, y)$ between two point $x, y$ was replaced by a real function $F_{x y}$ whose value $F_{x y}(t)$, for any positive real $t$, is interpreted as the probability

Received: August 7, 2015

(C) 2015 Academic Publications, Ltd.

${ }^{\S}$ Correspondence author url: www.acadpubl.eu 
that the distance between $x$ and $y$ is less than or equal to $t$. The study of these spaces was performed extensively by B.Schweizer and A.Sklar [9].

We briefly recall some definitions and known results about probabilistic metric spaces, for more detail we refer the reader to [9], Recall that a nonnegative real function $f$ defined on $\overline{\mathbb{R}^{+}}=\mathbb{R}^{+} \cup\{+\infty\}$ is known as a distance distribution function (briefly, a d.d.f) if it is non-decreasing, left continuous on ] $0,+\infty[$, with $f(0)=0$ and $f(+\infty)=1$. The set of all d.d.f will be denoted by $\Delta^{+}$.

A simple example of distribution function is Heavyside function defined by:

$$
H(t)= \begin{cases}0 & \text { if } t \leq 0 \\ 1 & \text { if } t>0\end{cases}
$$

In the sequel we will define some functions, on $\mathbb{R}^{+}$and consider them automatically extended to $\overline{\mathbb{R}^{+}}$.

According to [9] a commutative, associative and non-decreasing mapping $\tau: \Delta^{+} \times \Delta^{+} \rightarrow \Delta^{+}$is called a triangle function if and only if $\tau(f, H)=f$ for all $f \in \Delta^{+}$.

A triangle function $\tau$ is called sup-continuous, if for any family $\left\{F_{i}: i \in I\right\}$ of $\Delta^{+}$and any $G \in \Delta^{+}$we have $\tau\left(\sup _{i \in I} F_{i}, G\right)=\sup _{i \in I} \tau\left(F_{i}, G\right)$.

Definition 1.1. Let $f$ and $g$ be in $\Delta^{+}$, let $h$ be in $(0,1]$, and let $(f, g ; h)$ denote the condition

$$
0 \leq g(x) \leq f(x+h)+h \quad \text { for all } \quad x \in\left(0, \frac{1}{h}\right)
$$

The modified Lévy distance is the function $d_{L}$ defined on $\Delta^{+} \times \Delta^{+}$by

$$
d_{L}(f, g)=\inf \{h: \operatorname{both}(f, g ; h) \text { and }(g, f ; h) \text { hold }\} .
$$

Lemma 1.1. ([9])

(1) The pair $\left(\Delta^{+}, d_{L}\right)$ is a compact metric space.

(2) For any $t>0$

$$
f(t)>1-t \quad \text { if and only if } \quad d_{L}(f, H)<t
$$

(3) If $f$ and $g$ are in $\Delta^{+}$and $f \leq g$. Then $d_{L}(g, H) \leq d_{L}(f, H)$

Definition 1.2. By a probabilistic metric space we mean a triplet $(M, F, \tau)$ such that $M$ is nonempty set, $\tau$ is a triangle function and $F$ is a mapping from $M \times M$ into $\Delta^{+}$satisfying the following conditions for all $p, q, r$ in $M$ : 
(i) $F_{p p}=H$,

(ii) $F_{p q} \neq H$ if $p \neq q$,

(iii) $F_{p q}=F_{q p}$;

(iv) $F_{p q} \geq \tau\left(F_{p r}, F_{r q}\right)$.

For a given PM space $(M, F, \tau)$, B. Schweizer and A.Sklar ([9]) point out that If $\tau$ is continuous, then $F$ generates a first countable Hausdorff topology $\mathcal{T}_{F}$ on $M$ which has as a base the family of sets of the form $\left\{N_{x}(t): x \in M, t>0\right\}$, where $N_{x}(t)=\left\{y \in M: F_{x y}(t)>1-t\right\}$. On the other hand $\left\{U_{t}: t>0\right\}$ is a base for a uniformity $\mathcal{U}(F)$ on $M$ compatible with $\mathcal{T}_{F}$, where $U_{t}=\{(x, y) \in$ $\left.M \times M: F_{x y}(t)>1-t\right\}$ for all $t>0 . \mathcal{U}(F)$ is called the strong uniformity induced by $F$. In the sequel, when we speak about a PM space $(M, F, \tau)$, we always assume that $\tau$ is continuous.

In virtue of $\mathcal{T}_{F}$, a sequence $\left(x_{n}\right)_{n \in \mathbb{N}}$ in $M$, is said to be convergent to $x$ (we write $x_{n} \rightarrow x$ or $\left.\lim _{n \rightarrow \infty} x_{n}=x\right)$ if

$$
\forall t>0 \exists n_{0} \in \mathbb{N} \quad \text { such that } n \geq n_{0} \Rightarrow F_{x_{n} x}(t)>1-t,
$$

A sequence $\left(x_{n}\right)_{n \in \mathbb{N}}$ in $M$ is said to be Cauchy if

$$
\forall t>0 \exists n_{0} \in \mathbb{N} \quad \text { such that } n, m \geq n_{0} \Rightarrow F_{x_{n} x}(t)>1-t,
$$

The PM space $(M, F, \tau)$ is said to be complete, if every Cauchy sequence in $M$ converges to some point in $M$.

Following [10] (see also, [9]) a completion of a PM space $(M, F, \tau)$ is a pair $((N, G, \sigma), \Phi)$, where $(N, G, \sigma)$ is a complete $\mathrm{PM}$ space and $\Phi$ is an isometry from $M$ into $N$ (i.e. $F_{p q}=G_{\Phi(p) \Phi(q)}$ for all $p, q \in M$ ) such that $\Phi(M)$ is dense in $N$. It is well known that every metric space has a completion which is unique up to isometry see for instance [4]. Sherwood in [10] established the following result (see also [9])

Lemma 1.2. ([9],[10]) Every PM space $(M, F, \tau)$ with a continuous triangle function has a completion that is unique up to isometry.

Indeed, The construction of the completion of a $\mathrm{PM}$ space $(M, F, \tau)$ with continuous triangle function follows the line as in the classical metric case.

In 1968 R.J. Egbert [3] extended the classical Hausdorff distance to the probabilistic setting by introducing the notion of probabilistic Hausdorff metric in the case of Menger space. Thereafter, Tardiff [11] enlarged this notion to the context of general probabilistic metric spaces (see also [9]). Let $(M, F, \tau)$ 
be a PM space and denote by $\mathcal{P}_{0}(M)$ the family of all nonempty subsets of $M$. Given $p \in M, B \in \mathcal{P}_{0}(M)$. "The probabilistic distance" form $p$ to $B$ is defined as

$$
F_{p B}(t)=F_{B p}(t)=\sup _{q \in B} F_{p q}(t) \quad \text { for all } t \in[0,+\infty)
$$

Given $A, B \in \mathcal{P}_{0}(M)$ and defined

$$
\mathcal{H}_{F}^{-}(A, B)(t)=\left\{\begin{array}{l}
0 \text { if } t=0 \\
\sup _{0<s<t} \inf _{p \in A} F_{p B}(s) \text { if } t \in(0,+\infty)
\end{array}\right.
$$

and

$$
\mathcal{H}_{F}^{+}(A, B)(t)=\left\{\begin{array}{l}
0 \text { if } t=0 \\
\sup _{0<s<t} \inf _{q \in B} F_{A q}(s) \text { if } t \in(0,+\infty)
\end{array}\right.
$$

The probabilistic Hausdorff distance between $A$ and $B$ is the d.d.f:

$$
\mathcal{H}_{F}(A, B)=\min \left\{\mathcal{H}_{F}^{-}(A, B), \mathcal{H}_{F}^{+}(A, B)\right\}
$$

In [9] it was proved that If the triangle function $\tau$ is sup-continuous. Then the triplet $\left(\mathcal{C}_{0}(M), \mathcal{H}_{F}, \tau\right)$ is a PM space, where $\mathcal{C}_{0}(M)$ denote the collection of nonempty closed subset of $M$. Moreover $\mathcal{H}_{F}$ satisfies the following properties, where $c l_{\mathcal{T}_{F}}$ denotes the closure of with respect to the strong topology.

Lemma 1.3. ([9],[2],[6]) Let $(M, F, \tau)$ a $P M$ space. Let $p, q \in M$ and $A, B \in \mathcal{P}_{0}(M)$. Then we have the following

(1) $x \in c l_{\mathcal{T}_{F}}(A)$ if and only if $F_{x A}=H$.

(2) $\mathcal{H}_{F}(\{p\},\{q\})=F_{p q}$ for all $p, q \in M$.

(3) $F_{p B} \geq \mathcal{H}_{F}^{-}(A, B) \geq \mathcal{H}_{F}(A, B)$.

(4) $\mathcal{H}_{F}^{+}(A, B)=\mathcal{H}_{F}^{-}(B, A), \mathcal{H}_{F}(A, B)=\mathcal{H}_{F}\left(c_{\mathcal{T}_{F}}(A), \operatorname{cl}_{\mathcal{T}_{F}}(B)\right)$, and $\mathcal{H}_{F}(A, B)=$ $H$ if and only if $c l_{\mathcal{T}_{F}}(A)=\operatorname{cl}_{\mathcal{T}_{F}}(B)$.

(5) If $\mathcal{H}_{F}(A, B)(t)>1-t$ for some $t>0$ then

$$
\begin{array}{ll}
\forall p \in A \quad \exists q \in B \quad \text { such that } & F_{p q}(t)>1-t \\
\forall q \in B \quad \exists p \in A \quad \text { such that } & F_{p q}(t)>1-t
\end{array}
$$

(6) If $\tau$ is sup-continuous then $F_{p B} \geq \tau\left(F_{p A}, \mathcal{H}_{F}(A, B)\right)$ 


\section{Main Results}

We begin this section by studying completeness property of the probabilistic Hausdorff metric. The first result is a direct consequences of [[6],Th 12].

Theorem 2.1. A PM space $(M, F, \tau)$ with sup-continuous triangle function is complete if and only if the PM space $\left(\mathcal{C}_{0}(M), \mathcal{H}_{F}, \tau\right)$ is complete.

To state our next result we recall that a subset $Y$ of an uniform space $(X, \mathcal{U})$ is said to be totally bounded if for every $U \in \mathcal{U}$ there exists a finite subset $Z$ of $X$ such that $Y \subset U(Z)=\bigcup_{z \in Z} U(z)$. where $U(z)=\{x \in X:(x, z) \in U\}$. A subset $Y$ of a uniform space $(X, \mathcal{U})$ is compact if and only if $Y$ is complete and totally bounded ([[5], Ch. 6, Th. 32]). Let $\mathcal{K}_{0}(M)$ denote the collection of nonempty compact subset of PM space $(M, F, \tau)$.

Theorem 2.2. A PM space $(M, F, \tau)$ with sup-continuous triangle function is complete if and only if PM space $\left(\mathcal{K}_{0}(M), \mathcal{H}_{F}, \tau\right)$.

Proof. At first suppose that $\left(\mathcal{K}_{0}(M), \mathcal{H}_{F}, \tau\right)$ is a complete PM space. Let $\left(x_{n}\right)_{n \in \mathbb{N}}$ be a Cauchy sequence in $M$ and consider the sequence $\left(A_{n}\right)_{n \in \mathbb{N}}$ where $A_{n}=\left\{x_{n}\right\}$ for all $n \in \mathbb{N}$. Then, from Lemma $1.3(2)\left(A_{n}\right)_{n \in \mathbb{N}}$ is Cauchy sequence in $\left(\mathcal{K}_{0}(M), \mathcal{H}_{F}, \tau\right)$, so there exists $A \in \mathcal{K}_{0}(M)$ such that $A_{n} \rightarrow A$ with respect to $\mathcal{H}_{F}$. it is easy to show that $\left(x_{n}\right)_{n \in \mathbb{N}}$ converge to every $x \in A$. hence $(M, F, \tau)$ is complete.

Conversely, by Theorem $2.1\left(\mathcal{C}_{0}(M), \mathcal{H}_{F}, \tau\right)$ is a complete PM space. Since $\mathcal{K}_{0}(M) \subset \mathcal{C}_{0}(M)$, it is enough to prove that $\mathcal{K}_{0}(M)$ is closed in $\mathcal{C}_{0}(M)$ with respect to $\mathcal{T}_{\mathcal{H}_{F}}$. Indeed, Let $\left(A_{n}\right)_{n \in \mathbb{N}} \subset \mathcal{K}_{0}(M)$ such that $A_{n}$ converge to $A \in \mathcal{C}_{0}(M)$ with respect to $\mathcal{T}_{\mathcal{H}_{F}}$. Let $t>0$, since $\tau$ is uniformity continuous, then there is $\mu_{t}$ such that for all $G, K \in \Delta^{+}$

$$
d_{L}\left(G, \varepsilon_{0}\right)<\mu_{t} \quad \Rightarrow \quad d_{L}(\tau(K, G), K)<\frac{t}{2} .
$$

The convergence of $\left(A_{n}\right)_{n \in \mathbb{N}}$ and (2) of Lemma 1.1 implies that there exists $N \in \mathbb{N}$ such that

$$
d_{L}\left(\mathcal{H}_{F}\left(A_{N}, A\right), H\right)<\frac{t}{2} .
$$

Now, let $p \in A$, by (5) of Lemma 1.3 and (2.2) of Lemma 1.1, there is $q \in A_{N}$ such that

$$
d_{L}\left(F_{p q}, \varepsilon_{0}\right)<\frac{t}{2}
$$


since $A_{N}$ is compact, so $A_{N}$ is totally bounded, Then there exists a finite subset $Z \subset M$ such that $A_{N} \subset U_{\mu t}(Z)$, which implies the existence of $z \in Z$ such that

$$
d_{L}\left(F_{q z}, \varepsilon_{0}\right)<\mu_{t}
$$

Hence form Lemma 1.1 (1), (2) and the inequalities (2.1), (2.3) and (2.4) we have

$$
\begin{aligned}
d_{L}\left(F_{p z}, \varepsilon_{0}\right) & \leq d_{L}\left(\tau\left(F_{p q}, F_{q z}\right), \varepsilon_{0}\right) \\
& \leq d_{L}\left(\tau\left(F_{p q}, F_{q z}\right), F_{p q}\right)+d_{L}\left(F_{p q}, \varepsilon_{0}\right) \\
& <\frac{t}{2}+\frac{t}{2}=t
\end{aligned}
$$

This shows that $A \subseteq U_{t}(Z)$. Thus, $A$ is totally bounded and since $A$ is closed in the complete $\mathrm{PM}$ space $(M, F, \tau)$, it follows that $A$ is complete. Hence, $A \in \mathcal{K}_{0}(M)$.

In the sequel we will refer to $(\widetilde{M}, \widetilde{F}, \tau)$ as the completion of the PM space $(M, F, \tau)$. Our next object is to find the relationship between $\left(\widetilde{\mathcal{C}_{0}(M)}, \widetilde{\mathcal{H}_{F}}, \tau\right)$ and $\left(\mathcal{C}_{0}(\widetilde{M}), \mathcal{H}_{\widetilde{F}}, \tau\right)\left(\operatorname{resp},\left(\widetilde{\mathcal{K}_{0}(M)}, \widetilde{\mathcal{H}_{F}}, \tau\right)\right.$ and $\left.\left(\mathcal{K}_{0}(\widetilde{M}), \mathcal{H}_{\widetilde{F}}, \tau\right)\right)$.

Theorem 2.3. Let $(M, F, \tau)$ be a PM space with sup-continuous triangle function. Then, $\left(\mathcal{C}_{0}(\widetilde{M}), \mathcal{H}_{\widetilde{F}}, \tau\right)$ and $\left(\widetilde{\mathcal{C}_{0}(M)}, \widetilde{\mathcal{H}_{F}}, \tau\right)$ are isometric.

Proof. Let $(M, F, \tau)$ be a PM space and consider the mapping $\Phi$ from $\mathcal{C}_{0}(M)$ into $\mathcal{C}_{0}(\widetilde{M})$ defined by $\Phi(A)=\operatorname{cl}_{\mathcal{T}_{\widetilde{F}}}(A)$ for all $A \in \mathcal{C}_{0}(M)$. We will claim that $\Phi$ is an isometry from $\left(\mathcal{C}_{0}(M), \mathcal{H}_{F}, \tau\right)$ to $\left(\mathcal{C}_{0}(\widetilde{M}), \mathcal{H}_{\widetilde{F}}, \tau\right)$. Let $A, B \in \mathcal{C}_{0}(M)$, from Lemma 1.3 (4) we have for all $t>0$

$$
\begin{aligned}
\mathcal{H}_{\widetilde{F}}(\phi(A), \phi(B))(t) & =\mathcal{H}_{\widetilde{F}}(A, B)(t) \\
& =\min \left\{\sup _{s<t} \inf _{a \in A} \sup _{b \in B} \widetilde{F}_{a b}(s), \sup _{s<t} \inf _{b \in B} \sup _{a \in A} \widetilde{F}_{b a}(s)\right\} \\
& =\min \left\{\sup _{s<t} \inf _{a \in A} \sup _{b \in B} F_{a b}(s), \sup _{s<t} \inf _{b \in B} \sup _{a \in A} F_{b a}(s)\right\} \\
& =\mathcal{H}_{F}(A, B)(t)
\end{aligned}
$$

In the next step we show that $\phi\left(\mathcal{C}_{0}(M)\right)$ is dense in $\mathcal{C}_{0}(\widetilde{M})$. Equivalently we need to show that

$$
\forall A \in \mathcal{C}_{0}(\widetilde{M}), \forall t>0 \quad \exists B \in \phi\left(\mathcal{C}_{0}(M)\right) ; \mathcal{H}_{\widetilde{F}}(A, B)(t)>1-t
$$


Let $A \in \mathcal{C}_{0}(\widetilde{M}), t>0$ and $0<s<t$. Since $\tau$ is uniformly continuous there is $\mu_{s}>0$ such that for all $G, K \in \Delta^{+}$

$$
G\left(\mu_{s}\right)>1-\mu_{s} \text { and } K\left(\mu_{s}\right)>1-\mu_{s} \quad \Rightarrow \quad \tau(G, K)(s)>1-s .
$$

Putting $t_{1}=\min \left\{s, \mu_{s}\right\}$, since $M$ is dense in $\widetilde{M}$, then for each $a \in A$ there exists $x_{a} \in M$ such that $\widetilde{F}_{a x_{a}}\left(t_{1}\right)>1-t_{1}$. Putting $B=\left\{x_{a}: a \in A\right\}$ and $C=c l_{\mathcal{T}_{F}}(B)$, we will show that $\mathcal{H}_{\widetilde{F}}(A, \phi(C))(t)>1-t$, i.e. $\mathcal{H}_{\widetilde{F}}(A, C)(t)>1-t$ by Lemma 1.3 (5). To this end take $a \in A$ then there exists $x_{a}$ such that $\widetilde{F}_{a x_{a}}\left(t_{1}\right)>1-t_{1}$, so $\widetilde{F}_{a C}\left(t_{1}\right) \geq \widetilde{F}_{a x_{a}}\left(t_{1}\right)>1-t_{1}>1-t$, then $\inf _{a \in A} \widetilde{F}_{a C}\left(t_{1}\right)>1-t$. Hence we conclude that

$$
\mathcal{H}_{\widetilde{F}}^{-}(A, C)(t)>1-t .
$$

Next, let $c \in C$, then there exists $x_{a} \in B$ such that $F_{c x_{a}}\left(t_{1}\right)>1-t_{1}$ and $a \in A$ with $\widetilde{F}_{x_{a} a}\left(t_{1}\right)>1-t_{1}$. Then we have

$$
\widetilde{F}_{c x_{a}}\left(\mu_{s}\right) \geq \widetilde{F}_{c x_{a}}\left(t_{1}\right)>1-t_{1} \geq 1-\mu_{s}
$$

and

$$
\widetilde{F}_{x_{a} a}\left(\mu_{s}\right) \geq \widetilde{F}_{x_{a} a}\left(t_{1}\right)>1-t_{1} \geq 1-\mu_{s}
$$

Combining (2.5) with (2.7) and (2.8), we obtain

$$
\widetilde{F}_{c A}(s) \geq \widetilde{F}_{c a}(s) \geq \tau\left(\widetilde{F}_{c x_{a}}, \widetilde{F}_{x_{a} a}\right)(s)>1-s>1-t .
$$

Consequently

$$
\mathcal{H}_{\widetilde{F}}^{-}(C, A)(t)>1-t
$$

Hence,from (2.6) and (2.9) we conclude that $\mathcal{H}_{\widetilde{F}}(A, C)(t)>1-t$. This shows that $\phi\left(\mathcal{C}_{0}(M)\right)$ is dense in $\mathcal{C}_{0}(\widetilde{M})$ and since by Theorem $2.1\left(\mathcal{C}_{0}(\widetilde{M}), \mathcal{H}_{\widetilde{F}}, \tau\right)$ is a complete PM space, we deduce that $\left(\mathcal{C}_{0}(\widetilde{M}), \mathcal{H}_{\widetilde{F}}, \tau\right)$ is a completion of $\left(\mathcal{C}_{0}(M), \mathcal{H}_{F}, \tau\right)$. Thus, by Theorem 1.2 we conclude that $\left(\mathcal{C}_{0}(\widetilde{M}), \mathcal{H}_{\widetilde{F}}, \tau\right)$ and $\left(\widetilde{\mathcal{C}_{0}(M)}, \widetilde{\mathcal{H}_{F}}, \tau\right)$ are isometric.

Now we will show that Theorem 2.3 remain valid if we replace $\mathcal{C}_{0}(M)$ by $\mathcal{K}_{0}(M)$.

Lemma 2.1. Let $(M, F, \tau)$ be a $P M$ space with sup-continuous triangle function. If $K$ is a dense subset of $M$, then $\mathcal{K}_{0}(K)$ is dense in the PM space $\left(\mathcal{K}_{0}(M), \mathcal{H}_{F}, \tau\right)$. 
Proof. Let $A \in \mathcal{K}_{0}(M), t>0$ and $0<s<t$. Since $\tau$ is uniformly continuous, then there is $\mu_{s}>0$ such that for all $G, R \in \Delta^{+}$

$$
G\left(\mu_{s}\right)>1-\mu_{s} \text { and } R\left(\mu_{s}\right)>1-\mu_{s} \Rightarrow \tau(G, R)(s)>1-s .
$$

Putting $t_{1}=\min \left\{s, \mu_{s}\right\}$. Since $\mathrm{A}$ is compact, then $A$ is totally bounded, so there exists a finite subset $Y=\left\{y_{1}, \ldots, y_{n}\right\}$ such that $A \subset \underset{1 \leq i \leq n}{\bigcup} U_{t}\left(y_{i}\right)=$ $\bigcup_{1 \leq i \leq n} N_{y_{i}}(t)$.By hypothesis $K$ is dense in $M$, then for each $i \in\{1, \ldots, n\}$ there exists $z_{i} \in K$ such that $F_{z_{i} y_{i}}\left(t_{1}\right)>1-t_{1}$. Putting $Z=\left\{z_{1}, \ldots, z_{n}\right\}$. Applying the same arguments as in Theorem 2.3 we show that $\mathcal{H}_{F}^{-}(Z, A)(t)>1-t$ and $\mathcal{H}_{F}^{-}(A, Z)(t)>1-t$ hence we conclude that $\mathcal{H}_{F}(A, Z)(t)>1-t$.

Theorem 2.4. Let $(M, F, \tau)$ be a $P M$ space with sup-continuous triangle function. Then, $\left(\mathcal{K}_{0}(\widetilde{M}), \mathcal{H}_{\widetilde{F}}, \tau\right)$ and $\left(\widetilde{\mathcal{K}_{0}(M)}, \widetilde{\mathcal{H}_{F}}, \tau\right)$ are isometric.

Proof. Using the fact the restriction to $\mathcal{K}_{0}(M)$ of the map $\Phi$ defined in Theorem 2.3 is the identity, Lemma 2.1 and Theorem 2.2 we deduce that $\left(\mathcal{K}_{0}(\widetilde{M})\right.$, $\left.\mathcal{H}_{\widetilde{F}}, \tau\right)$ is a completion of $\left(\mathcal{K}_{0}(M), \mathcal{H}_{F}, \tau\right)$. The conclusion follows immediately from Theorem 1.2.

\section{Relative Results}

In this section we will show a counterpart of Theorem 2.3 and Theorem 2.4 in the case of metric spaces. In the sequel $(\widetilde{M}, \widetilde{d})$ denote the completion of given metric space $(M, d)$. It is well known (see, [1]) that for a given metric space $(M, d)$ the Hausdorff metric on the collection af all closed subsets $\mathcal{C}_{0}(M)$ is defined as the infinite valued metric $\mathcal{H}_{d}: \mathcal{C}_{0}(M) \times \mathcal{C}_{0}(M) \rightarrow[0, \infty]$ by $\mathcal{H}_{d}(A, B)=$ $\max \left\{\mathcal{H}_{d}^{-}(A, B), \mathcal{H}_{d}^{+}(A, B)\right\}$, where

$$
\mathcal{H}_{d}^{-}(A, B)=\sup _{a \in A} d(a, B) \text { and } \mathcal{H}_{d}^{+}(A, B)=\sup _{b \in B} d(A, b)
$$

In [8] Michael proved that a metric space $(M, d)$ is complete if and only if $\left(\mathcal{C}_{0}(M), \mathcal{H}_{d}\right)$ is complete.

Let $(M, d)$ be a metric space and consider the mapping $F^{d}: M \times M \rightarrow \Delta^{+}$ defined by

$$
F_{p q}^{d}(t)=\frac{t}{t+d(p, q)} \quad \text { for all } p, q \in M, t>0
$$


It is easy to check that $\left(M, F^{d}, \pi\right)$ is a PM space where $\pi(f)=,f . g$ for all $f, g \in \Delta^{+}$and that $\left(M, F^{d}, \pi\right)$ is complete if and only if $(M, d)$ is complete. In addition we have

Lemma 3.1. Let $(M, d)$ be a metric space then

(i) $\mathcal{H}_{F^{d}}$ and $F^{\mathcal{H}_{d}}$ coincide in $\mathcal{C}_{0}(M)$.

(ii) $\widetilde{F}^{d}$ and $F^{\widetilde{d}}$ coincide in $\widetilde{M}$.

Proof. (i) Let $A, B \subset \mathcal{C}_{0}(M)$ and $s>0$, we can easily show that $F_{p B}^{d}(s)=$ $\frac{s}{s+d(p, B)}$, then $\inf _{a \in A} F_{a B}^{d}(s)=\frac{s}{s+\sup _{a \in A} d(a, B)}$. Thus

$$
\mathcal{H}_{F^{d}}^{-}(A, B)(t)=\sup _{s<t} \frac{s}{s+\sup _{a \in A} d(a, B)}=\frac{t}{t+\mathcal{H}_{d}^{-}(A, B)}(t)=F_{A B}^{\mathcal{H}_{d}^{-}}(t)
$$

similarly we show that $\mathcal{H}_{F^{d}}^{+}(A, B)=F_{A B}^{\mathcal{H}_{d}^{+}}$. Hence, $\mathcal{H}_{F^{d}}=F^{\mathcal{H}_{d}}$.

(ii) Let $\widetilde{p}, \widetilde{q} \in \widetilde{M}$ and $\left(p_{n}\right)_{n \in \mathbb{N}},\left(q_{n}\right)_{n \in \mathbb{N}}$ two Cauchy sequences in $(M, d)$ such that $\left(p_{n}\right)_{n \in \mathbb{N}} \in \widetilde{p}$ and $\left(q_{n}\right)_{n \in \mathbb{N}} \in \widetilde{q}$ Then for each $t>0$ we have $\widetilde{F}_{\widetilde{p}}^{d}(t)=$ $\lim _{n} F p_{n} q_{n}(t)=\frac{t}{t+\lim _{n} d\left(p_{n}, q_{n}\right)}=\frac{t}{t+\widetilde{d}(\widetilde{p}, \widetilde{q})}=F_{\widetilde{p} \widetilde{q}}^{\widetilde{d}}(t)$. Hence, $\widetilde{F}^{d}$ and $F^{\widetilde{d}}$ coincide in $\widetilde{M}$.

Theorem 3.1. Let $(M, d)$ be a metric space. Then

(i) $\left(\mathcal{C}_{0}(\widetilde{M}), \mathcal{H}_{\widetilde{d}}\right)$ and $\left(\widetilde{\mathcal{C}_{0}(M)}, \widetilde{\mathcal{H}_{d}}\right)$ are isometric.

(ii) $\left(\mathcal{K}_{0}(\widetilde{M}), \mathcal{H}_{\widetilde{d}}\right)$ and $\left.\left(\widetilde{\mathcal{K}_{0}(M)}\right), \widetilde{\mathcal{H}_{d}}\right)$ are isometric.

Proof. From Lemma 3.1 we have $\mathcal{H}_{\widetilde{F}}=\mathcal{H}_{F \widetilde{d}}=F^{\mathcal{H}_{\widetilde{d}}}$ on $\mathcal{C}_{0}(\widetilde{M})$ and $\widetilde{\mathcal{H}_{F}}=\widetilde{F^{\mathcal{H}_{d}}}=F^{\widetilde{\mathcal{H}_{d}}}$ on $\widetilde{\mathcal{C}_{0}(M)}$. from Theorem 2.3 there is and isometry $\Phi$ from $\left(\mathcal{C}_{0}(\widetilde{M}), F^{\mathcal{H}_{\widetilde{d}}}\right)$ into $\left(\widetilde{\mathcal{C}_{0}(M)}, F^{\widetilde{\mathcal{H}_{d}}}\right)$. hence $\Phi$ is an isometry from $\left(\mathcal{C}_{0}(\widetilde{M}), \mathcal{H}_{\widetilde{d}}\right)$ into $\left(\widetilde{\mathcal{C}_{0}(M)}, \widetilde{\mathcal{H}_{d}}\right)$. (ii) follows similarly by replacing Theorem 2.3 by Theorem 2.4 .

\section{References}

[1] G.Beer, Topologies on Closed and Closed Convex Sets, Kluwer Academic Publishers, Dordrecht (1993). 
[2] Ş.Cobzaş, Completeness with respect to the probabilistic PompeiuHausdorff metric, Stud. Univ. Babes-Bolyai Math., 52, No.3 (2007), 43-65.

[3] R.J. Egbert, Products and quotients of probabilistic metric spaces, Pacific J. Math., 24, (1968), 437-455.

[4] N.R. Howes, Modern Analysis and Topology, Springer-Verlag, New York, (1995).

[5] J.L.Kelley, General Topology, Van Nostrand, New York (1957).

[6] A.Mbarki, A.Oahab, R.Naciri, The Probabilistic Hausdorff Quasi-metric and Right K-Completeness, International Journal of Pure and Applied Mathematics, Accepted.

[7] K. Menger, Statistical metrics, Proc. Nat. Acad, of Sci., U.S.A., 28, (1942), 535-537.

[8] E.Michael, Topologies on spaces of subsets, Trans.Amer.Math.Soc., 71, (1951), 152-182.

[9] B. Schweizer and A.Sklar, Probabilistic Metric Spaces, Series in Probability and Applied Mathematics, North-Holland (1983).

[10] H.Sherwood, Complete probabilistic metric spaces, Z. Wahrsch. Verw. Gebiete., 20, (1971), 117-128.

[11] R.M. Tardiff, Topologies for probabilistic metric spaces, Pacific J. Math., 65, No.1 (1976), 233-251.

[12] JZ. Xiao, XH. Zhu, and J. Yan, Probabilistic fractals and attractors in Menger spaces, Nonlinear Anal., 97, (2014), 106-118. 